\title{
Methods for Measuring Laser Power
}

\author{
Lyubomir Lazov \\ Rezekne Academy of Technologies \\ Rezekne, Latvia \\ llazov@abv.bg
}

\author{
Tsanko Karadzhov \\ Technical University of Gabrovo \\ Gabrovo, Bulgaria \\ karadjov_st@abv.bg
}

\begin{abstract}
Today we are witnessing the rapid development of the laser industry. Laser sources with new wavelengths, higher powers and energies, different modes of operation (generation of laser pulses), as well as various applications in industry, medicine, environmental protection etc. are emerging. This requires the development of new physical methods and principles for accurate measurement of power, energy, and other parameters of laser sources. In different types of laser technological processes, accurate measurement of laser power is extremely important in terms of quality, repeatability, and validation of the process. In most cases, accurate laser power measurement is a difficult task, especially when working with high-power lasers or having to perform real-time measurements.

The report analyses and systematizes the physical principles and methods on which existing laser measuring instruments are based. This research also aims to help researchers and technologists find and develop new approaches to solving this challenging measurement problem.
\end{abstract}

Keywords - laser power, measurement methods, types of measuring devices, physical principles

\section{INTRODUCTION}

In recent years, the laser industry has developed extremely fast, with more new laser sources with new wavelengths, higher power and energy, frequencies, and modes of operation. All this is a prerequisite for the emergence of new applications in various fields of industrial production.

The result and the quality for each specific laser technology such as laser welding, cutting, marking, engraving, drilling, etc. They depend on many factors, including material properties, processing speed, type of shielded gas, laser parameters, including - laser power [1] [2].

Laser power or more precisely the power density together with the processing speed (respectively the exposure time) are two of the main factors determining the degree of energy absorbed in the material. Therefore, to characterize in a qualitative aspect a technological operation for qualification, it is necessary to specify/quantify the power delivered by the laser in the processing area.

As laser technology is significantly ahead of the methods for measuring the parameters of laser radiation, it is necessary in recent years to look for new opportunities for improvement and refinement of technologies for measuring the power / energy of laser sources. [3] [4] [5]

1. The system requirements for accurate power output or energy become higher. Years ago a tolerance of $\pm 10 \%$ of power was considered good, today the requirements are higher to $\pm 5 \%$, and in some cases even higher \pm $1 \%$. Today, some pulsed laser applications require pulsed power stability of up to $0.2 \%$.

2. Lasers have become not only more powerful, but with versatile applications. For example, lasers are available on the market emitting $300 \mathrm{~J} / \mathrm{s}$ or $10 \mathrm{~kW}$ of pulsed power in the processing area, the energy density and power reaches values of the order of several hundred $\mathrm{J} / \mathrm{m}^{2}$ or over $10 \mathrm{~kW} / \mathrm{cm}^{2}$. For such power densities, new constructions and methods for measuring the energy parameters of the laser are needed.

Today, power and energy meters have to deal with increasingly difficult and difficult tasks due to the fact that they have to measure large intervals of change in power, energy (from power $\mathrm{pW}$ up to $10 \mathrm{~kW}$; pJ energy to hundreds of J), the length of the wave ( from far UV to far IR), the diameter of the beam, for example the pulse duration and the repetition rate. An example of this great variety of measuring devices offered on the market of different types, operating ranges and based on different methods, can be seen from just one portfolio of one of the world's leading companies Coherent [6]. 
TABLE 1

\begin{tabular}{|c|c|c|c|c|}
\hline $\begin{array}{l}\text { Laser } \\
\text { Type }\end{array}$ & $\begin{array}{l}\text { Measurement } \\
\text { Needed }\end{array}$ & $\begin{array}{l}\text { Power } \\
\text { Range }\end{array}$ & $\begin{array}{c}\text { Wavelength } \\
\text { Range }\end{array}$ & $\begin{array}{l}\text { Sensor } \\
\text { Type }\end{array}$ \\
\hline $\mathrm{CW}$ & Avg Power & $\begin{array}{l}10 \mathrm{nW} \text { to } \\
50 \mathrm{~mW} \\
200 \mu \mathrm{WW} \text { to } \\
>5 \mathrm{~kW}\end{array}$ & $\begin{array}{l}250 \mathrm{~nm} \text { to } \\
1800 \mathrm{~nm} \\
0.15 \mu \mathrm{m} \text { to } \\
12 \mu \mathrm{m}\end{array}$ & $\begin{array}{l}\text { Optical - } \\
\text { Power Mode } \\
\text { Thermopile }\end{array}$ \\
\hline Pulsed & Avg Power & $\begin{array}{c}200 \mu \mathrm{W} \text { to } \\
>5 \mathrm{~kW}\end{array}$ & $\begin{array}{c}0.15 \mu \mathrm{m} \text { to } \\
12 \mu \mathrm{m}\end{array}$ & Thermopile \\
\hline Pulsed & $\begin{array}{l}\text { Energy Per } \\
\text { Pulse }\end{array}$ & $\begin{array}{c}100 \mathrm{~nJ} \text { to } \\
>10 \mathrm{~J}\end{array}$ & $\begin{array}{l}0.15 \mu \mathrm{m} \text { to } \\
12 \mu \mathrm{m}\end{array}$ & Pyroelectric \\
\hline Pulsed & $\begin{array}{l}\text { Energy Per } \\
\text { Pulse }\end{array}$ & $\begin{array}{c}10 \mathrm{pJ} \text { to } 800 \\
\mathrm{~nJ}\end{array}$ & $\begin{array}{l}325 \text { to } 1700 \\
\mathrm{~nm}\end{array}$ & $\begin{array}{l}\text { Optical - } \\
\text { Energy } \\
\text { Mode }\end{array}$ \\
\hline
\end{tabular}

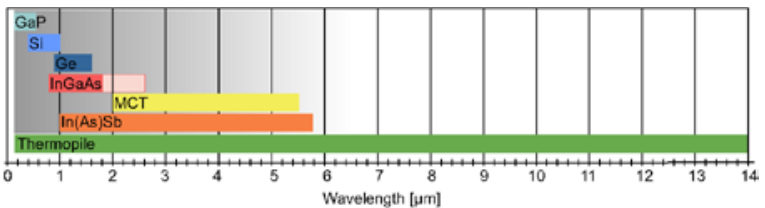

Fig. 1. The sensitivity of two types of sensors (photodiode detectors and thermopiles) for measuring power relative to the wavelengths of laser radiation [7]

When choosing a meter for the power of laser radiation, it is necessary to take into account two factors, the method of measurement and the range of operation of the sensor. The importance of this parameter for two spacing measurement methods is sowed by the diagram in Figure 2.

Table 1 Different types of measuring devices offered by Coherent with their operating ranges of: power and wavelengths.Measurement and monitoring of laser power is a continuous process from the beginning - the moment when the laser is first manufactured, to its integration and operation by the end-user, who will use the laser system in various types of applications that we know are so wide from industrial applications to those in medicine and research. The choice of the appropriate measuring equipment for the work in the specific technological applications is no less important than the choice of the appropriate laser for the realization of a specific technological process.

Currently, available laser measuring devices use different measuring methods and sensors. The choice of a specific meter for a given application depends on the spectral range, sensitivity or minimum required response and trigger threshold.

The most commonly used methods for measuring laser power are given in the following table2.

TABLE 2

\begin{tabular}{|c|c|}
\hline Method & Features and specifics of the method \\
\hline $\begin{array}{l}\text { with } \\
\text { pyroelectric } \\
\text { sensors }\end{array}$ & $\begin{array}{l}\text { - Measures the energy of pulsed lasers and } \\
\text { can only work with pulsed lasers } \\
\text { - The average power is calculated by } \\
\text { multiplying the pulse repetition frequency by } \\
\text { the pulse energy. }\end{array}$ \\
\hline $\begin{array}{l}\text { with thermopile } \\
\text { sensor }\end{array}$ & $\begin{array}{l}\text { - Measures the power of CW lasers and } \\
\text { integrates the energy of pulsed lasers to } \\
\text { determine the average measured power. } \\
\text { - It can also be used to measure the energy } \\
\text { of a pulse - most often to measure the energy } \\
\text { of medical and industrial lasers with wide } \\
\text { pulses. }\end{array}$ \\
\hline $\begin{array}{l}\text { with optical } \\
\text { sensors - } \\
\text { Photodiode }\end{array}$ & $\begin{array}{l}\text { - Used to measure low CW laser power. } \\
\text { - It is also used in some sensors to measure } \\
\text { low-pulsed laser power. }\end{array}$ \\
\hline
\end{tabular}

The variety of sensitivity ranges of different types of sensors operating in different circuit solutions and serving concrete methods of power measurement are also in a wide range. Figure 1 presents the possible ranges of operation of two types of sensors used in thermal and optical methods. The graph shows that thermopile sensors cover a wider range than optical / diode laser radiation sensors.

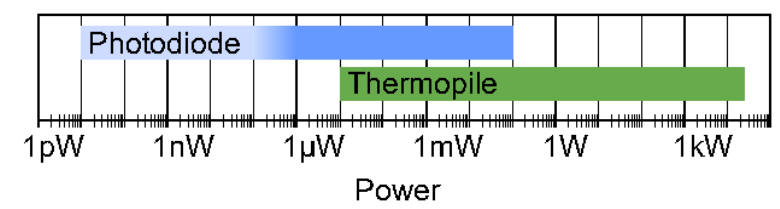

Fig. 2. Operating ranges of laser power measuring devices based on two different methods using photodiode and thermopile detectors [7].

Both types of detectors cover a large dynamic range of powers. It is clear from the figure that devices based on the thermal method are preferred when measuring high laser power powers of the order of $\mathrm{kW}$, and photoelectric methods based on diode sensors are preferred at low powers in the range $\mathrm{pW}$ to $\mathrm{mW}$.

Some several key steps and considerations need to be taken in the specific selection of the measuring device. This is because, as there are many different types of lasers with very different specifications, also there are different measurement technologies that are suitable for specific types of lasers and tailored to specific laser specifications. In this article, we will try to discuss the basic approaches and methods of measuring the power and energy of the laser and we will try to give you a small contribution to the selection process faced by each user of laser technology equipment.

\section{THERMAL MEASUREMENT METHODS}

The thermal method using thermopile sensors is a great universal technology suitable for measuring the power of many lasers. They can be successfully used to measure the power of CW lasers as well as the average power of pulsed lasers. In some special cases, they can also be used to measure the energy of individual long pulses. The basic physical principle on which this type of power meter has based the conversion of the absorbed electromagnetic energy of the laser into heat. With the help of a heat radiator, this heat is transferred to the environment by convective cooling or water cooling. The temperature difference between the temperature of the sensor/absorber and the radiator/cooler is converted into an electrical signal by a thermocouple.

Photodiodes are sensitive for powers down to $10 \mathrm{pW}$. Incoming radiation above $100 \mathrm{~mW}$ leads to a saturation of the electrical signal, setting the limit for the maximum 
power. The power saturation is critical when monitoring e.g., ultrafast pulse lasers. Although the average power might be rather low (and within the power range of the detector), the power of the short pulses greatly exceeds the upper power limit of the detector leading to saturation of the signal and hence distorted measurement results. Additionally, industrial applications use photodiodes typically from 500nW upwards (when detecting stray light or fractions of the beam), giving a very small dynamic range and complicating its integration into the system. For such applications, a thermopile detector is preferable, since it has a broad dynamic range and integrates the signal of the individual pulses, therefore monitoring the average power.

Thermopile sensors are used to monitor laser beams of up to $25 \mathrm{~kW}$. The upper limit for those detectors is mainly attributed to the ability to cool the sensor efficiently. In recent years, the minimum detectable power has been shifted more and more into the range of photodiodes and has reached $10 \mu \mathrm{W}$. The company greenTEG offers a resolution below $1 \mu \mathrm{W}$. [7]

\section{Measurement of laser radiation power with thermocouple}

Thermopile sensors are the best choice for measuring average powers from $10 \mu \mathrm{W}$ upwards. Especially outside the visible range, where photodiode technologies are expensive, thermopiles should be considered. Photodiodes, on the other hand, are the best choice when single pulses need to be analysed or when power levels in the nW range should be resolved.

Most power meters are based on the principle of a thermal detector: They convert optical power to heating power via an absorber structure with a black coating. They use a thermopile to measure the resulting temperature rise (or the temperature difference between the absorber and the mount). Thermal power meters are useful for average powers between about $10 \mathrm{~mW}$ and several kilowatts. Thermal power meters are moderately precise, have a sensitivity that is independent of wavelength, and are relatively slow.

In many cases, these meters use thermocouples. The measured laser beam falls on one side of the thermocouple, it is heated by the laser, and on the other side, there is a radiator or cooler, which aims to maintain a constant temperature. The laser energy absorbed by the thermocouple material is converted into heat. The temperature difference on both sides of the thermocouple creates an electromotive voltage. This voltage is proportional to the temperature difference, which is proportional to the measured power. on the laser. This voltage is measured to provide laser power readings in watts fig. 3 .

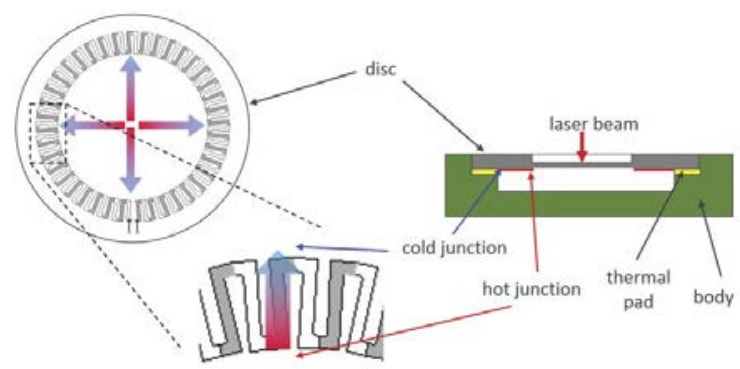

Fig. 3. Disk with thermocouple [8]

The material used to absorb the energy of the laser beam is essential because the parameters and characteristics of the detector depend on its properties. This material absorbs most of the laser's optical energy and converts it into heat. A portion of the laser beam is reflected, which can vary from a few percent to 50 percent of the total optical power, depending on the material.

The laser beam falls in the center of the disk and the heat energy passes radially from the center to the periphery. The total amount of heat passing through the disk does not depend on the size of the disk and the position of the beam.

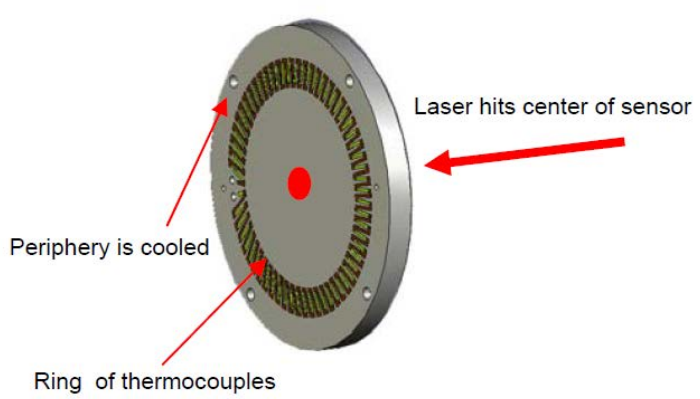

Fig. 4. Thermocouples

The thermal power meter is not measuring the absolute temperature of the sensor but rather the temperature drops across the thermopile fig. 4 . Therefore, the reading is quite independent of ambient temperature. If the temperature of the cooled periphery goes up, the temperature of the inside of the thermopile goes up correspondingly and the temperature drop - hence the reading - remains the same.

Thermopile laser measurement is almost wavelengthindependent, durable, and reliable. It is therefore the mainstay of laser power/energy measurement. It cannot, however, measure repetitive pulses or very low powers and energies.

\section{Pyroelectric power meters}

The pyroelectric sensor used in laser power measuring devices consists of a crystal that polarizes when heated. These detectors work as follows:

When a laser pulse hits the surface of the detector, it heats up and polarizes the crystal, thus creating an even and opposite charge on both surfaces of the detector. The surface of the detector is metallized so that the charge 
collects on the parallel capacitor, regardless of where the laser beam hits the surface. In this way, the charge of the capacitor is proportional to the energy of the pulse. Once the pulse is complete, the capacitor voltage is read, and the capacitor is discharged electronically to be ready for the next pulse.

Pyroelectric detectors are especially useful for repetitive pulses and can measure up to thousands of pulses per second. They are also quite sensitive. However, they are not very durable, so for higher energies and powers, an attenuator/diffuser is placed in front of the sensor crystal to reduce the energy on the pyroelectric crystal.

Pyroelectric power meters are relatively fast meters. They cannot be used to measure the continuous power of the laser. The output signal of this type of laser power meter is proportional to the changes in the incident input energy.

Pyroelectric sensors for measuring radiant energy are sensitive in a very wide range of wavelengths and can measure energy from laser sources with different powers up to $10 \mathrm{~J}$. Most of these sensors have a ceramic coating to withstand high energy density of laser radiation. The sensors can be connected directly to a high input impedance amplifier or oscilloscope via a BNC connector.

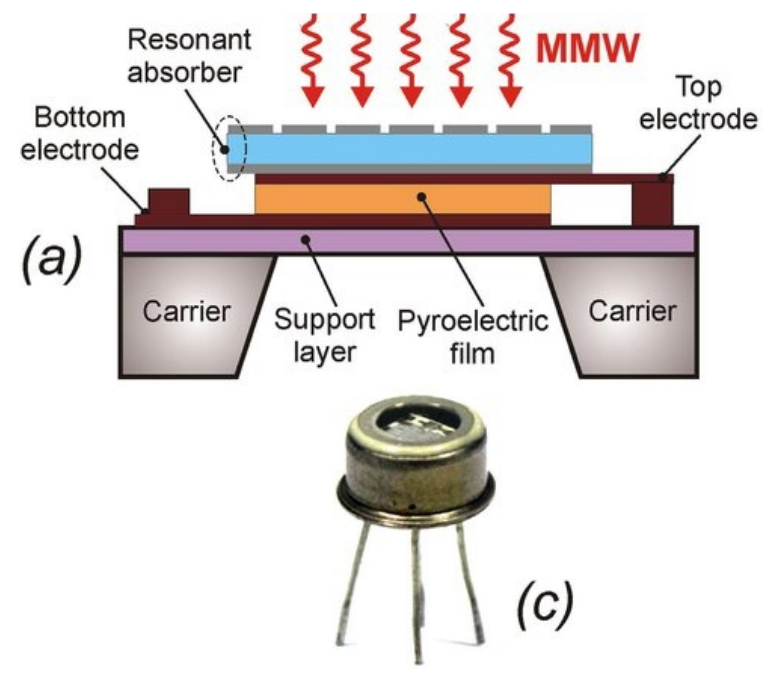

Fig. 5. Pyroelectric sensor [9]

\section{PHOTOELECTRIC MEASUREMENT METHODS}

The most commonly used sensors for measuring low laser power are photodiodes due to their speed. In them, as in other sensors, light is absorbed by materials and converted into an electric current called photocurrent, which is proportional to the power of the radiation. Depending on the material from which they are made, photodiodes are sensitive to laser beams with different wavelengths. Photodiodes made of Si, Ge and InGaAs are sensitive in the near infrared region up to $1800 \mathrm{~nm}$. Using materials such as HgCdTe (MCT) and In (As) Sb, the measurement range is extended to about $6000 \mathrm{~nm}$. Photodiodes made of $\mathrm{GaP}$ are used to measure the power of ultraviolet lasers.
The principle of measuring with photodiodes is one of the most common methods for measuring the power of laser radiation. It has both advantages and some significant disadvantages. The action of the photodiode is based on increasing the reverse current of the p-n junction during its illumination, which is practically used to measure the intensity or power of laser radiation.

The photodiode has the highest speed of all optoelectronic photodetectors, which is its biggest advantage. It can be used to measure fast-changing laser radiation. The photodiode can operate in two modes photodiode and photo galvanic mode, and both modes can be used to measure the intensity of light or laser fluxes. Socalled PIN photodiodes are used to measure the power of pulsed lasers, which have an even higher speed than traditional photodiodes with a PN junction.

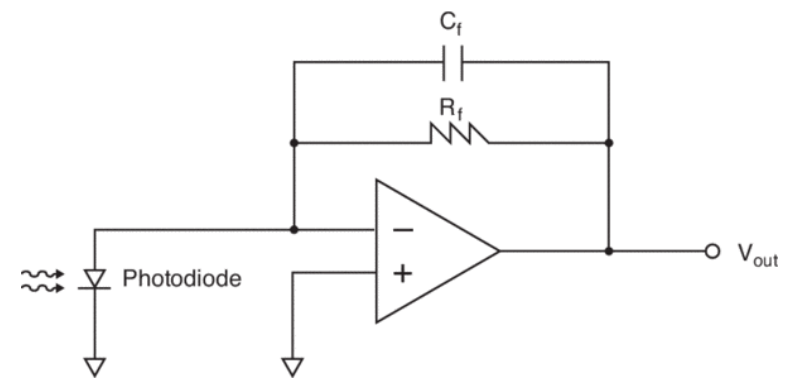

Fig. 6. Light-to-voltage converter

Most laser power measurement circuits use light to voltage converter at which the output voltage is proportional to the luminance over the active area of the photodiode created by the laser beam fig. 6 .

There are also power meters that use light to frequency converter with a photodiode at the input. This is a programmable light to frequency IC that outputs a pulse train or a square wave (50\% duty) with the frequency that is directly proportional to the incident light intensity. Uses a simple TTL output for easy interfacing to a microcontroller.

For example, the S9705 is a photo IC that combines a photodiode and a current-to-frequency converter on a CMOS chip. Output is a square wave (50 \% duty ratio) with frequency directly proportional to light intensity incident on the photodiode. The CMOS level digital output allows direct connection to a microcontroller or other logic circuitry. The S9705 has a wide dynamic range and light intensity can be easily measured when used with a digital counter [10].

Features

Converts light intensity to frequency

Wide dynamic range: 5 orders of magnitude

Excellent linearity

Output timing reset function

Digital output for direct interface to microcontroller 4-pin plastic package.

In rare cases, semiconductor phototransistors and photoresistors can be used as photosensitive elements. 


\section{PONDEROMOTIVE ACTION MEASUREMENT METHODS}

Another method is based on the physical phenomenon of optical radiation pressure. In this method, the measurement of the laser beam power is realized without the need to absorb it, as is necessary, for example, in the calorimetric method. There is no need for thermal control of the absorbed laser energy and the reaction and recovery time after each measurement in the receiver.

The idea of the radiation pressure or the momentum of photons was first proved by the Russian scientist Peter Nikolaevich Lebedev in 1899 [11]. Figure 1 shows a schematic diagram of the idea of the experiment proposed by Lebedev.

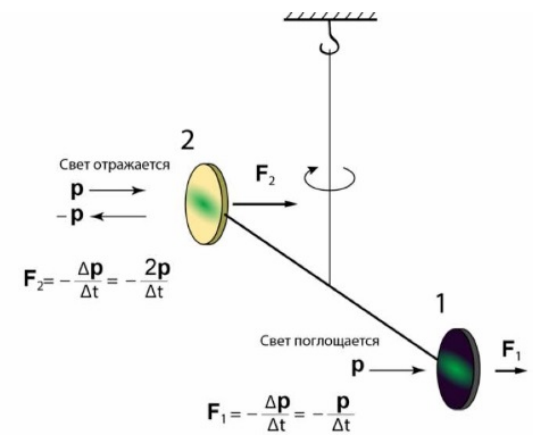

Fig. 7. Lebedev's experiment

It consists of two circular plates, one of which is blackened and absorbs light, and the other, shiny, reflects light fig. 7. The plates are fixed at opposite ends of a light rod suspended by a thin quartz thread. The whole structure was placed in a glass vessel from which air was evacuated. When reflected from a mirror, light transmits to it twice as much pulse per unit time as light falling on an absorbing plate of the same area, which leads to twisting of the filament, which was measured by P.N. Lebedev. It is worth noting that the actual installation was somewhat more complicated since it was necessary to avoid the effects associated with an insufficiently high vacuum level and uneven heating of the installation parts.

The light flux propagating in space carries with it a certain momentum, when interacting with a substance it transmits its momentum, changing its direction of propagation. For example, if the momentum carried by a light beam is $\mathrm{p}$, then the force $\mathrm{F}$ transmitted by it to the substance (mirror) depends on the rate of change of this pulse.

$$
F=\frac{d p}{d t}
$$

Since the magnitude of the pulse is defined by the amount of energy $\mathrm{E}$ divided by the speed of light c.

$$
|p|=\frac{E}{c}
$$

Then for a beam with optical power $P=d E / d t$, reflected from without diffusion and absorption by an ideal mirror will reflect the radiation of incident light, generating a force $F=2 P / c$ on the mirror. Thus, the light falling on a mirror with perfect reflection will press the mirror with force fig.8:

$$
F=\frac{2 P}{c} r \cos \theta
$$

where $\mathrm{r}$ is a function of the reflectivity $\mathrm{R}$ and the absorption $\mathrm{A}=(1-\mathrm{R})$, as well as the $\alpha$ part of the un-reflected light absorbed by the mirror

$$
r=R+(1-R) \alpha / 2
$$

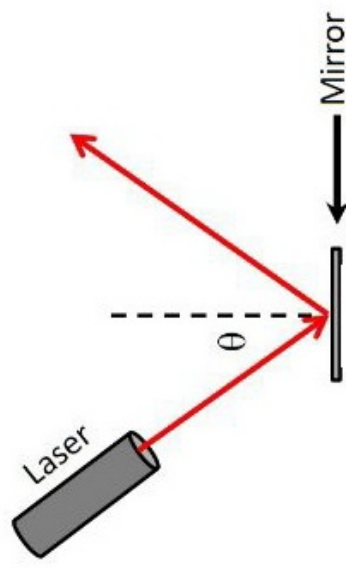

Fig. 8. The laser radiation, incident at $\theta$ angle to the mirror, exerts a force $\mathrm{F}$ on it.

Here the fact is considered that the absorbed photon gives its whole momentum, and the reflected photon gives twice its momentum to the mirror.

Equation (3) describes a maximum power conversion factor in force of $2 / c=6.67 \times 10^{-9} \mathrm{~N} / \mathrm{W}$ for a normal frequency of a perfectly reflecting mirror.

The first successful experiments demonstrating the light radiation pressure date back to the early 20th century [12] [13]. The presence of small forces at powers of the order of $100 \mathrm{~mW}$ of incident light on the plates of the scale is demonstrated using torsion scales.

These as well as later developments by many authors [14] [15] [16] [17] [18] [19] fully confirm the validity of the radiation pressure. This method with the use of a torsion balance has a high sensitivity but is not suitable for working in a non-laboratory environment. That is why it has been neglected for many years and can be said to have been forgotten.

Today, highly sensitive scales and dielectric mirrors with very high reflectivity are available (usually $>99.99 \%$ for optical and $>90 \%$ for RF). This is precisely the prerequisite for a new stage in the development of this method of measurement - mainly for measuring high laser power. Such devices are already available from several research laboratories. They are based on the physics of the interaction of incident photons on a strongly reflecting mirror [20]. The advantage of the device offered by these authors is the ability to directly deflect the laser beam from the measuring mirror, in which case the measurement is performed without interrupting the laser technology 
process itself. However, this proposed design solution has one significant drawback, namely sensitivity to inertial forces (acoustic vibrations and tilt-dependent gravity projection).

Ivan Ryger et al. [21] in their publication demonstrate a miniaturized version of a radiation pressure sensor that can potentially overcome the above-mentioned drawback, namely by avoiding the impact of the environment (acoustic vibrations and tilt-dependent gravity projection) on the sensor readings, thus closer to the needs of users of technological systems. A schematic diagram of the sensor proposed by the authors is shown in figure. 9. The sensor consists of a silicon micromachined parallel plate capacitor, both of whose disk electrodes (diameter $20 \mathrm{~mm}$ ) are attached to a rigid frame through weak springs (width $465 \mu \mathrm{m}$, thickness $380 \mu \mathrm{m}$, length $45 \mathrm{~mm}$ ) in the shape of an Archimedean spiral (see fig,9). For the optical sensor, a highly reflective dielectric mirror (optimized for reflectance with a value of $0.9992 \pm 0.0002$ at $1.07 \mu \mathrm{m}$ wavelength and $45^{\circ}$ angle of incidence) is deposited onto the front side of one disk electrode.

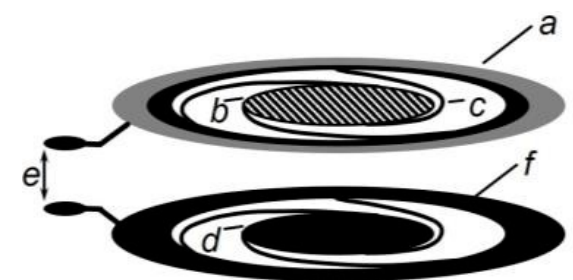

Fig. 9. Schematic diagram of a sensor for measuring laser radiation pressure using an Archimedean spiral for a damper. [22]

To obtain a calibration between the measured electrical signal and the applied optical radiation on the sensor, it is necessary to know exactly the electromechanical parameters (spring constant, the distance between the capacitor plates and the capacity gradient concerning the distance between the plates). For this purpose, Ivan Ryger et al. in [22] offers an optoelectronic characterization technique, where a time-varying voltage of electrostatic deviation is applied to the electrodes of the capacitor and measuring the axial displacement of the mirror.

\section{OTHER MEASUREMENT METHODS}

Piezo-resistance method for measuring laser radiation pressure

Piezoelectric sensors are based on the physical piezo effect. When charged with pressure on the piezoelectric materials on their opposite surfaces, electric charges appear. When the magnitude and direction of the pressure changes, the magnitude and type of electric charge also change fig. 10.

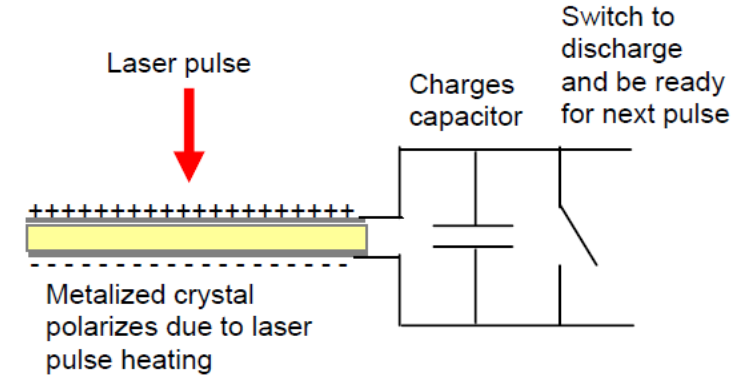

Fig. 10. Schematic diagram of a pressure sensor based on the piezoelectric effect

Piezo-resistance pressure sensors based on microelectro-mechanical systems (MEMS) on a silicon substrate can also be successfully used to measure the laser pressure. The sensor is made of a thin silicon membrane, hermetically covering the space below it, and four piezo resistors applied on its surface, the resistance of which depends on the pressure and temperature.

Any change in the external pressure under the action of laser radiation causes deformation of the membrane and a change in the resistance of the piezo resistors connected in a bridge circuit fig. 11 .
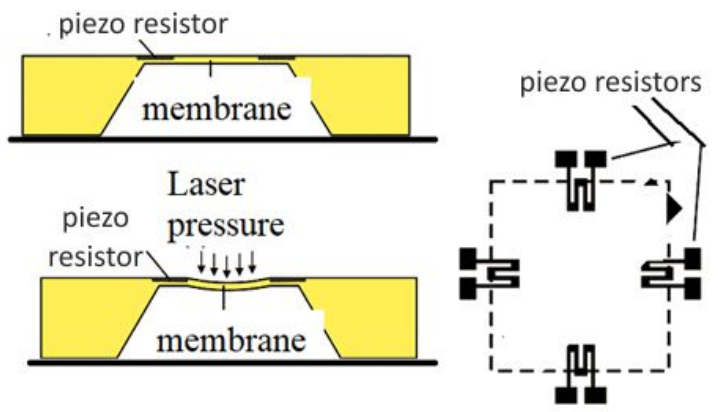

Fig. 11. Schematic diagram of membrane type pressure sensor in combination with piezo resistors

Nonlinear optical method effects for measuring the energy (power) of a laser radiation.

A method for measuring the energy or power of laser radiation, based on nonlinear optical effects arising from the passage of laser radiation through a substance, the main of which are the effect of optical rectification, the effect of optical polarization, generation of harmonics and Raman scattering.

Photochemical method for measuring the energy (power) of a laser radiation.

A method for measuring the energy or power of laser radiation based on the use of photochemical reactions with a known quantum yield arising from absorption laser radiation by substance.

Photographic method for measuring the energy (power) of a laser radiation.

A method for measuring the energy or power of laser radiation, based on the photochemical effect of laser radiation on photographic materials and functional dependence the optical density of blackening $D$ of the 
photosensitive layer from the irradiation of this layer $E$ and the exposure time $t$, determined by the formula:

$$
D=\left[\lg \left(E . t^{p}\right)\right]
$$

where $D$ is the optical density of blackening; $\mathrm{E}$ is the irradiance of the photosensitive layer; $t$ is the exposure time; $\mathrm{p}$ is the Schwarzschild parameter depending on $E$ and t.

\section{CONCLUSION}

The present study aims to help users of laser equipment in the correct selection of a laser measuring system. From all that has been said so far, the first step must begin with an analysis of a detailed examination of the laser (or lasers), the parameters of which must be measured:

Key laser parameters in this analysis should be minimum and maximum average power of the laser source, the wavelength of the laser radiation, and the output diameter of the laser radiation.

When working with a pulsed laser, it is important to consider: the minimum and maximum pulse energy as well as the width of the laser pulse and the frequency of repetition of the pulses. (If the repetition rate is adjustable, it will be useful to know the maximum repetition rate at maximum energy, thus obtaining the maximum average number of powers.) Taking these parameters and factors into account in the mode of operation of the laser will help to determine how much heat will be absorbed/absorbed if a sensor system is selected.

The following considerations/guidelines should be considered when determining the appropriate measurement method for a laser technology system:

- Water cooling is used in all modern thermal sensors to measure powers greater than $300 \mathrm{~W}$. And at high powers of the order of kilowatts, a specialized highspeed water line is provided in most cases for cooling the sensor.

- Thermopile sensors are preferable to use when it is necessary to measure the power of pulsed lasers with a pulse repetition frequency higher than $10 \mathrm{kHz}$. If the frequency of the pulsed laser is less than $10 \mathrm{kHz}$, it is preferable to use a pyroelectric sensor.

- To measure the power of lasers operating at low power and emitting in the far infrared region of the electromagnetic spectrum, it is preferable to use devices based on the photoelectric method. Semiconductor photodiode sensors are often used, which have a good response to low light levels. This type of method and measurement sensors can be successfully applied to lasers operating in CW and pulsed mode, but with low average output power.

- The next step after selecting the appropriate sensor for a given measurement is to select a compatible meter with the required measuring range and the required sensitivity. This step is very important to perform quality registration and visualization of the measurement. Usually the signal from the sensor is converted from analog to digital signal on the measuring display. The measuring device can also perform digital signal processing and other correction algorithms, such as wavelength or temperature compensation.

\section{REFERENCES}

[1] N. Angelov, "Investigation the Influence of the Number of Repetition and Volumetric Energy Density on Laser Marking of Products," Journal of the Academy of Sciences and arts of the Republic of Srpska „Contemporary Materials, vol. V, no. 2, pp. 250-253, 2014, DOI: 10.7251/cm.v2i5.1738

[2] N. Angelov, "Determination of Working Intervals of Power Density and Frequency for Laser Marking on Samples from Steel HS18-0-1,” Proceedings of the Union of Scientists - Ruse, vol. 12, pp. 125-130, 2015.

[3] A. L. Cullen, “ Absolute power measurement at microwave frequencies,” Proc. IEEE , vol. 92, pp. 100-111, 1952.

[4] D. Ma, J. L. Garrett and J. N. Munday, "Quantitative measurement of radiation pressure on microcantilever in ambient environmnent,” Appl. Phys. Lett, vol. 106, 2015, DOI: 10.1063/1.4914003

[5] K. Agatsuma, D. Friedrich, S. Ballmer, G. DeSalvo, S. Sakata, E. Nishida and S. Kawamura, "Precise measurement of laser power using an optomechanical system,” Opt. Express, vol. 22, pp. 2013-2030, 2014, DOI: 10.1364/OE.22.002013

[6] [Online]. Available: https://www.coherent.com/measurementcontrol/main/laser-power-and-energy-meters.

[7] S. Dröscher, "White Paper: Technologies for Laser Powe monitoring," [Online]. Available: https://www.greenteg.com/template/userfiles/files/White\%20Pa per_Detector\%20Technologies\%20Laser\%20Power.pdf.

[8] [Online]. Available: https://www.newport.com/t/optical-powermeasurement.

[9] S. A. Kuznetsov, A. G. Paulish, M. Navarro-Cía and A. V. Arzhannikov, "Selective Pyroelectric Detection of Millimetre Waves Using Ultra-Thin Metasurface Absorbers," Scientific Reports, vol. 6, 16 Feb 2016, DOI: 10.1038/srep21079

[10] [Online].

Available: https://www.hamamatsu.com/us/en/product/type/S9705/index.h tml.

[11] P. N. Lebedev, "Experimental examination of light pressure," Ann. Phys, pp. 1-26, 1901.

[12] E. F. Nichols and G. F. Hull, "A preliminary communication on the pressure of heat and light radiation," Phys. Rev, vol. 13, p. 307-320, 1901, DOI: 10.1103/PhysRevSeriesI.13.307

[13] E. F. Nichols and G. F. Hull, "The pressure due to radiation," Astrophysical. Journal, vol. 17, pp. 315-351, 1903.

[14] J. J. Cook, W. L. Flowers and C. B. Arnold, "Measurement of laser output by light pressure,” Proc. IRE , vol. 50, 1962.

[15] M. Stimler, Z. I. Slawsky and R. E. Grantham, "Torsion pendulum photometer,” Rev. Sci. Instrum, vol. 35, pp. 311-313, 1964, DOI: $10.1063 / 1.1718813$

[16] G. Roosen and C. Imbert, "Measurement of laser output by light pressure,” Opt. Eng, vol. 20, pp. 437-439, 1981.

[17] G. T. Gillies, "Photomechanical laser power measurements with magnetically suspended rotors,” Fizika, vol. 19, pp. 407-420, 1987.

[18] Y. Yuan, “A new pulse laser energy meter,” Rev. Sci. Instrum, vol. 61, p. 1743-1746, 1990, DOI: 10.1063/1.1141144 
[19] G. T. Gillies and R. C. Ritter, "Torsion balances, torsion pendulums, and related devices,” Rev. Sci. Instrum, vol. 64, no. 2, p. 283, 1993, DOI: 10.1063/1.1144248

[20] P. Williams, J. A. Hadler, F. C. Maring, R. Lee, K. A. Rogers, B. J. Simonds, M. T. Spidell, A. D. Feldman and J. H. Lehman, "Portable, high-accuracy, non-absorbing laser power measurement at kilowatt levels by means," Opt. Express , vol. 25, p. 4382-4392, 2017.

[21] I. Ryger and et al, "MEMS Non-Absorbing Electromagnetic Power Sensor Employing the Effect of Radiation Pressure,” in
Proceeding at the Eurosensors , Graz, Austria, 2018, DOI: 10.3390/proceedings2130767

[22] I. Ryger, A. B. Artusio-Glimpse, P. Williams, N. Tomlin, M. Stephens, K. Rogers, M. Spidell and J. Lehman, "Micromachined force scale for optical power measurement by radiation pressure sensing,” IEEE Sens. J. , vol. 18, p. 79417948, 2018, DOI: 10.1364/OE.385502 